\title{
ANTIGENIC CROSS-REACTIVITY OF VENOMS OBTAINED FROM SNAKES OF GENUS BOTHROPS
}

\author{
A. M. Moura da Silva, ${ }^{1}$ M. R. D'Impèrio Lima, ${ }^{2}$ A. K. Nishikawa, ${ }^{3}$ C. I. Brodskyn, ${ }^{1}$ \\ M. C. dos Santos, ${ }^{2}$ M. F. D. Furtado, ${ }^{4}$ W. Dias da Silva, ${ }^{5}$ and I. Mota ${ }^{1}$ \\ 'Centro de Imunologia, Instituto Butantan, São Paulo, Brazil; '2Departamento de Imunologia, Instituto de \\ Ciências Biomédicas, Universidade de São Paulo, São Paulo, Brazil; '3ecção de Concentração de Soros, \\ Instituto Butantan, São Paulo, Brazil; ‘Seção de Venenos, Instituto Butantan, São Paulo, Brazil; \\ 'Laboratório Especial de Imunoquímica, Instituto Butantan, São Paulo, Brazil
}

(Accepted for publication 6 July 1989)

A. M. Moura da Silva, M. R. D’Impèrio lima, A. K. Nishikawa, C. I. Brodskyn, M. C. dos Santos, M. F. D. Furtado, W. Dias da Silva and I. Mota. Antigenic cross-reactivity of venoms obtained from snakes of genus Bothrops. Toxicon 28, 181-188, 1990.-Antigenic cross-reactivity was studied among the components of venoms from nine species of the genus Bothrops using species-specific antivenoms. Sera titration by DOT-ELISA detected similar levels of antibody when either homologous or heterologous antigens were used. Transblotted antigens, after SDS-PAGE fractionation, were also revealed by homologous and heterologous antivenoms. Antigens with mol. wt greater than 30,000 seemed to be the most cross-reactive. Antigens of about 24,000 mol. wt were poorly immunogenic. Antigens between $14-18,000 \mathrm{~mol}$. wt cross-reacted only with B.moojeni, B.jararacussu, B. neuwiedi and B. pradoi venoms. Neutralization of the lethality of $B$. jararaca venom was observed by homologous and heterologous antivenoms.

\section{INTRODUCTION}

SNAKE venoms consist of mixtures of numerous proteins with enzymatic and/or toxic activities. Venoms from snakes belonging to the same family contain components of very similar activities in spite of their heterogeneous electrophoretic patterns (GonçALVES and VIEIRA, 1950; GonÇAlves and DuETSCH, 1956). In Brazil, most envenomations are due to bites by snakes of the genus Bothrops. This genus includes several different species (HoGE and Romano-Hoge, 1978/1979). At the Institut Butantan, (São Paulo) bothropic antivenom is produced by immunization of horses with venom obtained from seven species (RoLIM-RosA et al., 1980/1981). This raises some questions about the efficacy of such antisera in cases of envenomation by snake species whose venom is not included in the pool of venoms used for immunization.

Several groups of researchers have already shown that the venom of each species can be partially or even totally neutralized by heterologous antisera (HousSAY and NeGRETE, 
1923; Minton, 1967; Tu and Salafranca, 1974; Bolanos et al., 1975). Double immunodiffusion tests have shown that these venoms share many common components (SILESVillarRoEl et al., 1974) including haemorrhagic (MaNDELBaUm and AsSaKuRA, 1988) and coagulant fractions (RosenFeLD and KELEN, 1966), which contribute to, this crossreactivity. However, some venom components are species-specific since they do not show antigenic cross-reactivity (SILES-VILLARROEL et al., 1974).

The present work reports the results of a comparative study of the antigenic composition of the venoms of nine different species of the genus Bothrops analyzed by DOTELISA and Western Blotting.

\section{MATERIAL AND METHODS}

One month old, female A/Sn mice weighing 22-25g (Instituto Butantan animal house) were used. B. alternatus, B. atrox, B. cotiara, B erythromelas, B. jararaca, B jararacussu, B. moojeni, B. neuwiedi and B. prado $i$ venoms were extracted from 30-40 adult snakes using standard procedures. Venoms of each species were pooled, dried and stored at $4^{\circ} \mathrm{C}$ before use.

For immunization $100 \mu \mathrm{g}$ of each venom was added to $1 \mathrm{ml}$ of a suspension containing $1 \mathrm{mg} / \mathrm{ml} \mathrm{Al}(\mathrm{OH})_{3}$ as adjuvant. The mixture was stirred for $15 \mathrm{~min}$ to allow adsorption of the antigen onto the adjuvant particles. Nine groups of 18 mice were injected i.p. with four doses of $200 \mu \mathrm{l}$ of each venom/adjuvant mixture at 7 day intervals. A control group received only $\mathrm{Al}(\mathrm{OH})_{3}$ on the same days as the experimental groups. Blood was collected 28 days after the last immunization dose from the brachial plexus of the animals under light ether anaesthesia. Sera were separated and stored at $-20^{\circ} \mathrm{C}$ until used.

For the antibody assay $2 \mu \mathrm{g}$ of each venom were dotted onto nitrocellulose strips (Trans-Blot, BIO-RAD, Laboratories, Richmond, CA, U.S.A.). After drying at room temperature, the nitrocellulose was blocked with 5\% dehydrated skimmed milk (Molico-Nestlé, São Paulo, Brazil) in Tris-buffered saline and then placed into incubation chambers containing titrated sera anti-specific and anti-heterologous Bothrops venom. After $1 \mathrm{hr}$ at room temperature, strips were incubated for $1 \mathrm{hr}$ with horseradish peroxidase-conjugate anti-mouse IgG (Cooper Biomedical Inc., Malvern, PA 19355, U.S.A.) diluted 1:1000 in Tris-buffered saline. The affinity staining for the horseradish peroxidase was performed with DAB $\left(3,3^{\prime}\right.$ diaminobenzidine, Sigma Chemical Company, St Louis, MO, U.S.A.) at a concentration of $250 \mu \mathrm{l}$ of the stored solution $(20 \mathrm{mg} / \mathrm{ml})$ in $30 \mathrm{ml}$ of Tris buffered saline in the presence of $30 \% \mathrm{H}_{2} \mathrm{O}_{2}(20 \mu \mathrm{l})$. Titres were visually determined (Table 1).

Venoms were analyzed by SDS polyacrylamide gel electrophoresis and Western blot using 8-18\% gradient polyacrylamide slab gels under non-reducing conditions. Samples $(80 \mu \mathrm{l})$ were boiled in SDS (5 min) at a concentration of $1000 \mu \mathrm{g} / \mathrm{ml}$. The gels were stained with Coomassie Brilliant Blue R-250 (BIO-RAD). Western blots were obtained by transblotting from the polyacrylamide gels onto nitrocellulose paper. The blots were blocked with $5 \%$ dehydrated milk in Tris buffered saline. Serum samples (1:100 dilutions) were added and were incubated for $1 \mathrm{hr}$ at room temperature. After washing with Tris buffered saline, the strips were incubated with peroxidase conjugate anti-mouse IgG (1:1000 dilution). The affinity staining was performed as described above.

TABLE 1. ANTIBOdY titers $\left(\times 10^{-3}\right)$ OF THE ANTTVENOM TESTED Aganst HOMOLOGOUS AND HETEROLOCOUS VENOMS AS ANTIGENS BY DOT-ELISA

\begin{tabular}{|c|c|c|c|c|c|c|c|c|c|}
\hline \multirow[t]{2}{*}{ Venoms } & \multicolumn{9}{|c|}{ Antivenoms against } \\
\hline & ALT & ATR & COT & ERY & JAR & JUS & MOO & NEU & PRA \\
\hline ALT & 512 & 256 & 128 & 128 & 128 & 64 & 128 & 256 & 128 \\
\hline ATR & 512 & 256 & 128 & 128 & 128 & 128 & 128 & 256 & 128 \\
\hline COT & 256 & 256 & 256 & 128 & 128 & 128 & 128 & 256 & 128 \\
\hline ERY & 512 & 256 & 128 & 256 & 128 & 128 & 128 & 256 & 128 \\
\hline JAR & 16 & 32 & 128 & 128 & 128 & 128 & 128 & 256 & 128 \\
\hline JUS & 64 & 128 & 64 & 32 & 32 & 128 & 64 & 128 & 64 \\
\hline MOO & 64 & 128 & 64 & 128 & 128 & 128 & 256 & 128 & 64 \\
\hline NEU & 64 & 64 & 64 & 128 & 128 & 128 & 128 & 128 & 64 \\
\hline PRA & 64 & 64 & 64 & 128 & 128 & 128 & 128 & 128 & 64 \\
\hline
\end{tabular}


Lactalbumin $(14,000)$, trypsinogen $(24,000)$, egg albumin $(43,000)$, bovine serum albumin $(68,000)$ and murine IgG $(150,000)$ were used as molecular weight markers.

For the neutralization test of lethality $B$.jararaca venom was mixed with antiserum to the venom of either $B$. jararaca, B. erythromelas, B. moojeni or serum of mice which received only adjuvant or saline. The mixtures were incubated for $1 \mathrm{hr}$ at $37^{\circ} \mathrm{C}$ and then injected i.p. into groups of eight mice. The individual dose was $70 \mu \mathrm{g}$ of venom $\left(2 \mathrm{LD}_{90}\right)$ and $200 \mu \mathrm{l}$ of antiserum. The number of dead animals was assessed after 1, 2, 3, 4, $24 \mathrm{and} 48 \mathrm{hr}$. Animals were examined for peritoneal haemorrhagic lesions immediately after death or, in the case of surviving mice, $\mathbf{4 8} \mathrm{hr}$ after injection when they were killed.

\section{RESULTS}

\section{Cross titration of the species-specific antivenoms}

Antibodies present in species-specific antivenoms prepared in mice were estimated by the DOT-ELISA technique using homologous and heterologous venoms as antigens. Table 1 shows the antibody titres obtained against either homologous or heterologous antigens. Levels ranged from 1:64,000-1:512,000 and did not differ significantly. However, there were a few exceptions represented by the anti-B.alternatus and anti-B. atrox sera against $B$. jararaca venom and anti- $B$. atrox serum against $B$. jararacussu venom. In these cases, the antibody titres were respectively 32 and 16 fold lower than the titres of the homologous antisera.

\section{$S D S-P A G E$ and immuno blotting}

Figure 1 shows the distinct electrophoretic pattern of the nine venoms used for immunization. Figures 2, 3 and 4 show immuno blots of $B$. erythromelas, $B$. jararaca and $B$. moojeni venoms respectively, where the antigens were revealed with the nine speciesspecific antivenoms.

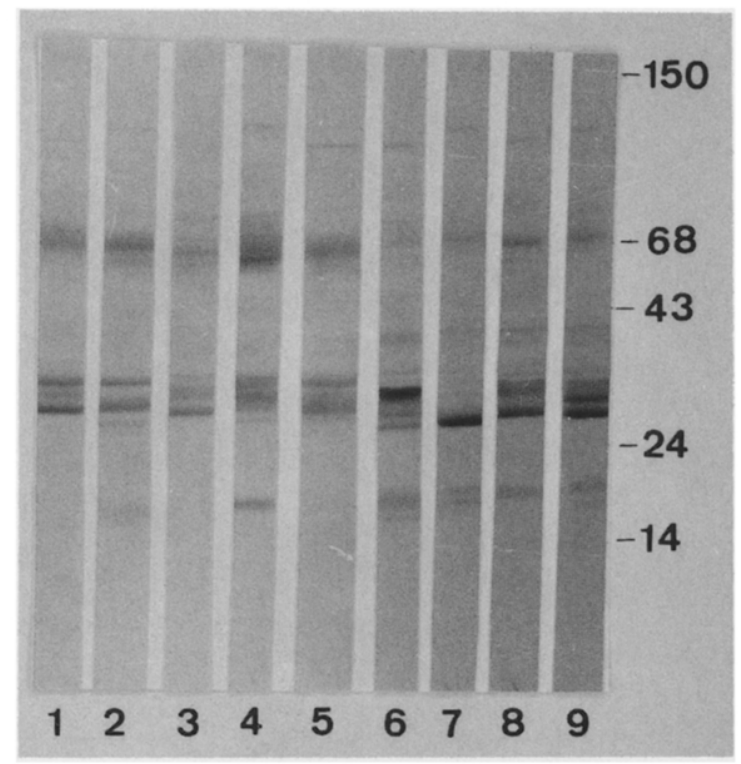

Fig. 1. VenOMS OF B. alternatus (1), B. atrox (2), B. cotiara (3), B. erythromelas (4), B. jararaca (5), B. jararacussu (6), B. moojeni (7), B. neuwiedi (8) AND B. pradoi (9) FRACTIONATED BY SDS-PAGE. Proteins were stained by Coomassie Blue. Mol.wt values are shown on the right. 


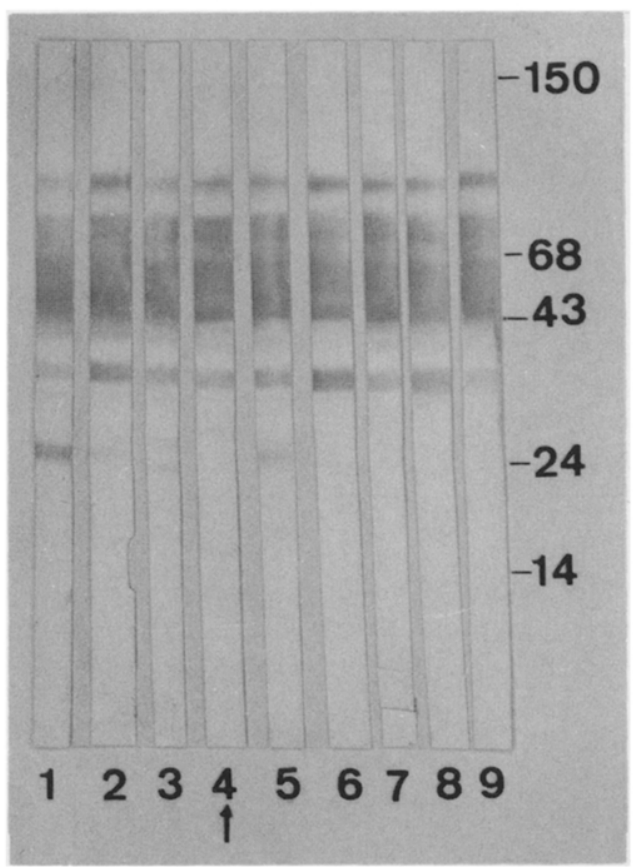

FIG. 2. ANTIGENS OF $B$. erythromelas VENOM WERE FRACTIONATED BY SDS-PAGE AND REVEALED BY WESTERN BLOTTING BY ANTI-B. alternatus (1), ANTI-B. atrox (2), ANT1-B. cotiara (3), ANTI-B. erythromelas (4), ANTI-B.jararaca (5), ANTI-B. jararacussu (6), ANT1-B. moojeni (7), ANTI-B. neuwiedi (8) AND ANTI-B. pradoi (9) SERA.

Fractionated components of B. erythromelas venom (Fig. 2) were recognized almost identically by all nine antisera. No difference could be detected in the antigens of mol. wt greater than 30,000 , except for the 42,000 band which was not detectable by the anti- $B$. jararacussu sera. However, each sera recognized different bands between 20 and 30,000 mol. wt. Thus, anti-B. alternatus intensely stained a band of 23,000 ; anti- $B$. atrox stained the same band weakly; anti-B. cotiara stained two bands with 29 and 21,000 ; anti-B. erythromelas stained only a 29,000 band while anti-B.jararaca stained 29 and $23,000 \mathrm{~mol}$. wt bands. No bands were stained in this region by the anti-B.jararacussu, anti-B. moojeni, anti-B. neuwiedi and anti-B. pradoi sera.

When $B$.jararaca venom was used as antigen (Fig. 3), the immuno blot pattern was slightly different for each antiserum. High mol. wt bands $(120,000)$ were stained only by $B$. jararaca, B. jararacussu, B. moojeni and B. neuwiedi antivenoms. B. erythromelas antivenom did not recognize the 66,000 antigen and $B$.jararacussu antivenom failed to recognize the 47,000 band. B. alternatus and $B$.pradoi antivenoms revealed the blots weakly. Most cross reactive antigens seemed to be located in the $48-60,000$ mol. wt region. Antigens of mol. wt in the region of 22,000 again showed a different pattern of recognition by each sera: anti-B. alternatus, anti-B. atrox and anti-B. jararaca stained a 21,000 band, anti-B. cotiara stained 25 and 21,000 bands. No band was stained in this region by the anti-B.erythromelas, anti-B.jararacussu, anti-B.moojeni, anti-B.neuwiedi and anti-B. pradoi.

B. moojeni venom showed a different electrophoretic pattern than B.jararaca and B. erythromelas venoms (Fig. 1). Blots stained with homologous sera (Fig. 4) showed that 


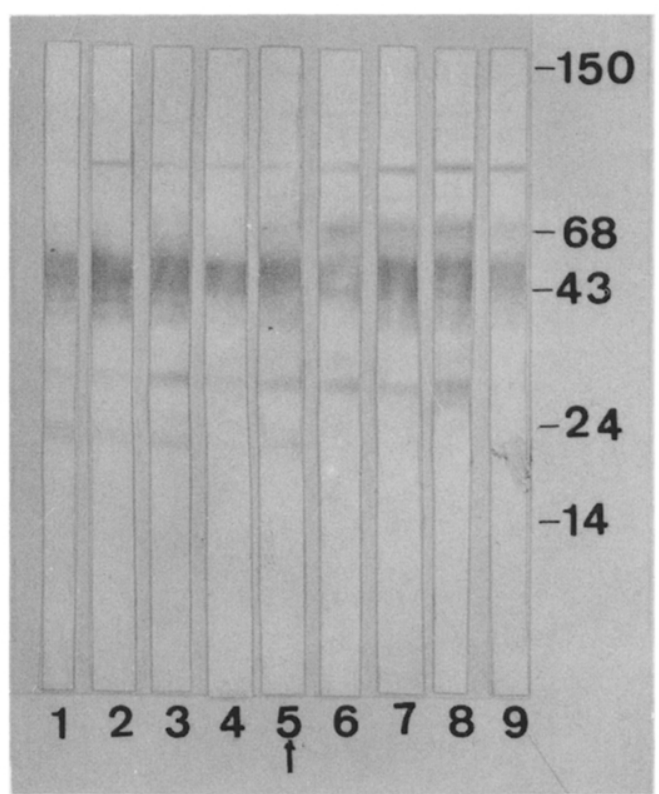

Fig. 3. ANTIGENS OF B. jararaca VENOM WERE FRACTIONATED BY SDS-PAGE AND REVEALED BY WESTERN BLOTTING BY ANTI-B.alternatus (1), ANTI-B.atrox (2), ANTI-B. cotiara (3), ANTI-B. erythromelas (4), ANTI-B. jararaca (5), ANTI-B.jararacussu (6), ANTI-B. moojeni (7), ANTI-B. neuwiedi (8) AND ANT1-B. pradoi (9) SERA.

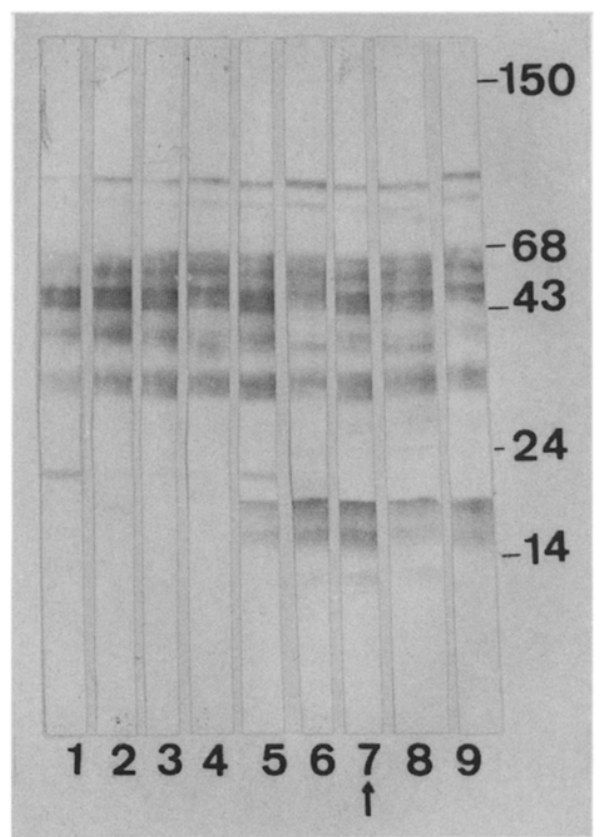

Fig. 4. ANTIGENS OF B. moojeni VENOM WERE FRACTIONATED by SDS-PAGE AND REVEALED BY WESTERN BLOTTING BY ANTI-B.alternatus (1), ANTI-B.atrox (2), ANTI-B. cotiara (3), ANTI-B. erythromelas (4), ANTI-B.jararaca (5), ANTI-B.jararacussu (6), ANT1-B.moojeni (7), ANTT-B. neuwiedi (8) AND ANTI-B. pradoi (9) SERA. 
the major antigens were located in the 17,000 region and between 28 and $60,000 \mathrm{~mol}$. wt. Many other bands could also be detected. Antigens with mol. wt greater than 31,000 were stained similarly by all nine sera. However, B. alternatus antivenom did not detect the 80 and 37,000 bands and the latter was also not stained by the B. atrox and B. cotiara antivenoms. The 22,000 band was stained strongly by $B$.alternatus and $B$.jararaca antivenoms and weakly by $B$.atrox and $B$.cotiara antivenoms. The most significant differences when $B$. moojeni venom was used was observed with antigens of mol. wt in the region of 14,000 . Bands of 14 and $18,000 \mathrm{~mol}$. wt were strongly stained only by $B$.jararaca, B.jararacussu, B.moojeni, B. neuwiedi and B.pradoi antivenoms and the 12,000 mol. wt band only by $B$.jararacussu, $B$. moojeni and $B$. neuwiedi antivenoms.

When $B$.alternatus, $B$. atrox and $B$. cotiara venoms were used as antigens they showed similar results to those obtained with the $B$.erythromelas blots. On the other hand, $B$. jararacussu, $B$. neuwiedi and $B$. pradoi venoms behaved similarly to $B$. moojeni (data not shown). Under our experimental conditions antigens recognized only by homologous serum were never detected.

\section{Neutralization experiments}

The large extent of antigenic cross reactivity observed in Western blotting suggests that each individual Bothrops venom could induce the production of antivenoms capable of protecting mice against the lethal effects of the other cross-reacting venoms. To test this, homologous and heterologous sera were reacted in vitro with the reference venom of B. jararaca for $1 \mathrm{hr}$ at $37^{\circ} \mathrm{C}$. The mixtures were subsequently injected in groups of mice. Figure 5 shows that groups of mice receiving $2 \mathrm{LD}_{50}$ of the venom mixed with saline or

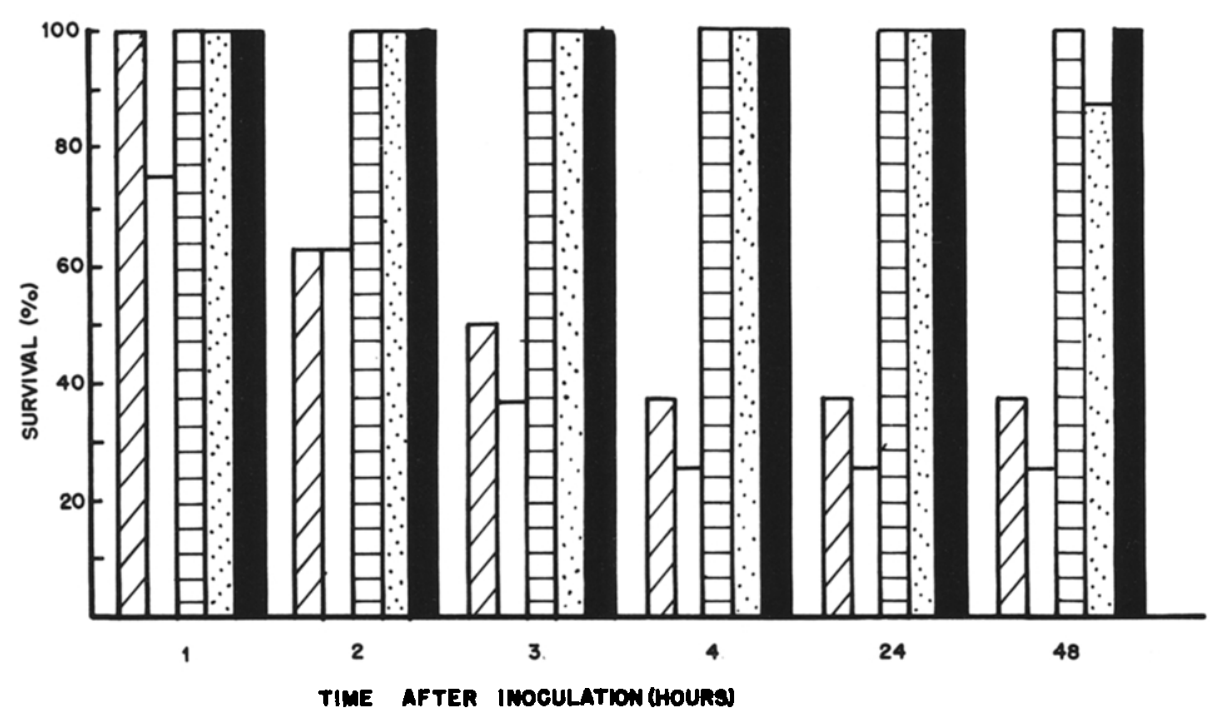

Fig. 5. DeCREASE IN THE LETHALITY INDUCED BY 2 LD $_{90}$ DOSES OF $B$. jararaca VENOM BY $200 \mu l$ OF EITHER ANTI-B. jararaca SERUM (国), ANTI-B. moojeni SERUM (团), ANTI-B. erythromelas SERUM ( $\square$ ), SERUM OF ANIMALS THAT RECEIVED ONLY ADJUVANT INJECTION (圆) OR SALINE ( $\square$ ).

Percentage of survived animals was determined $1,2,3,4,24$ and $48 \mathrm{hr}$ after inoculation. 
serum from animals injected only with adjuvant had survival rates of $38 \%$ and $25 \%$ respectively $4 \mathrm{hr}$ after injection. The survival rate did not decrease over $48 \mathrm{hr}$. However, mice that received the mixture containing $B$.jararaca venom plus its homologous or heterologous sera were strongly protected against the venom lethal effect as all the mice belonging to these groups survived for $48 \mathrm{hr}$, except one that received $B$. moojeni antivenom and died $48 \mathrm{hr}$ after injection. After $48 \mathrm{hr}$, all surviving animals were sacrificed and the peritoneal cavity was visually examined for haemorrhage. Mice which received $B$. moojeni and B.erythromelas antivenoms did not show signs of haemorrhage in the peritoneal cavity, while mice which received $B$.jararaca antivenom showed variable degrees of haemorrhage, especially at the site of injection. Dead mice all showed intense haemorrhage in the peritoneal cavity.

\section{DISCUSSION}

These results indicate a high degree of antigenic cross reactivity among the nine bothropic venoms studied. Seven of the venoms are from species occurring in the southern, central and southeastern regions of Brazil and are included in the pool of venoms used for immunization of horses at the Instituto Butantan for Bothrops antivenom production. B. atrox is the species of Bothrops most involved in ophidic accidents in the northern region of Brazil and $B$. erythromelas in the northeast. Thus, these venoms were included in order to study species from all the Brazilian regions.

In general, antigens with apparent mol. wt above 30,000 appeared to be the most crossreactive. This region of the gels includes the most immunogenic antigens as detected by titration of antivenoms specific for antigens separated by SDS-PAGE and transferred to nitrocellulose membranes (unpublished results). It is likely that these are the antigens mainly responsible for the antigenic cross reactivity detected earlier by double immunodiffusion (SILES-VillarRoel et al., 1974). This region also includes the haemorrhagic factor (MANDELBAUM et al., 1984), coagulant proteinase (GABRIJELCIC et al., 1982), thrombin-like enzymes (Kosugi et al., 1986) and other toxins. Proteins of $24,000 \mathrm{~mol}$. wt were strongly stained in all venoms by Coomassie Blue. In spite of this, they are weak immunogens in $B$. alternatus, B. atrox, B. cotiara, B. erythromelas and $B$. jararaca venoms, and non immunogenic in $B$. jararacussu, B. moojeni, B. neuwiedi and $B$. pradoi venoms.

Interesting results were observed for proteins in the region of $14-18,000 \mathrm{~mol}$. wt. The gel stained by Coomassie Blue, stressed bands in this region for B. atrox, B. erythromelas, B. moojeni, B. neuwiedi, B.jararacussu and B.pradoi venoms. However, they appeared only to be immunogenic in $B$.jararacussu, B. moojeni, B. neuwiedi and $B$. pradoi venoms. Bands belonging to the last four venoms may not represent the same antigens as the ones with the same mol.wt present in B.atrox and B.erythromelas venoms since in $B$. erythromelas and $B$. cotiara blots they were not stained by any antivenom. These bands are not present in $B$. jararaca venom; however anti- $B$. jararaca serum is able to recognize such antigens in B. moojeni (Fig. 3) and also in B. neuwiedi, B. jararacussu and B. pradoi blots (not shown). This strongly suggests the existence of epitopes shared by 14-18,000 mol. wt antigens of B. moojeni venom and other proteins of $B$. jararaca venom. The participation of these antigens in the toxic action of the venoms needs to be established. The presence of 14-18,000 mol. wt antigens only in B. jararacussu, B. moojeni, $B$. neuwiedi and $B$. pradoi venoms cannot be explained by similarity in geographic distribution since the four related species, $B$. alternatus, $B$. cotiara and $B$. jararaca occur also in the central-southern region of Brazil. On the other hand, phylogenetic differences among 
these species are poorly understood and the available data (BRATTSTROM, 1964) does not provide enough information to elucidate such data.

The most important point was that the high degree of antigenic cross reactivity observed in vitro could be confirmed in vivo since $B$. jararaca venom was neutralized similarly by homologous and heterologous sera. However, studies concerning cross neutralization of each toxic activity of the venoms must be carried out in order to clarify completely the antigenic cross reactivity of Bothrops venoms.

Acknowledgements-The authors are indebted to Mr CARLOS A. JARED for excellent technical assistance; Mr Marcelo Pedrosa Maestrelli for the invaluable help with the computer processing of our data and Dr R. D. G. Theakston for a critical review of the manuscript.

\section{REFERENCES}

Bolaños, R., Cerdas, L. and TAyLOR, R. (1975). The production and characteristics of a coral snake antivenom. Toxicon 13, 139-142.

BratTstrom, B. H. (1964) Evolution of the pit vipers. Trans. San Diego Soc. nat. Hist. 13, 185-268.

Gabrifelcic, D., Drujan, B. and Gubensek, F. (1982) Coagulation proteinase from Bothrops colombiensis venom. Toxicon 20, 275-278.

Gonçalves, J. M. and Deutsch, H. F. (1956) Ultracentrifugal and zone electrophoresis studies of some Crotalidae venoms. Archs Biochem. Biophys. 60, 402-411.

GONÇALVES, J. M. and VIERA, L. G. (1950) Estudos sobre venenos de serpentes brasileiras. l-Análise electroforètica. Anais Acad. bras. Ciênc. 32, 141-150.

Hoge, A. R. and Romano-Hoge, S. A. R. W. L. (1978/79) Sinopse das serpentes peçonhentas do Brasil. Mem. Inst. Butantan 42/43, 373-496.

Houssay, B. A. and Negrete, J. (1921) Proporciones en que el suero antiofidico neutraliza la ponzona de las serpientes. Revia. Asoc. méd. argent. 34, 845-861.

Kosugi, T., Ariga, Y., Nakamura, M. and Kinjo, K. (1986) Purification and some chemical properties of Thrombin-like enzyme from Trimeresurus flavoviridis venom. Thromb. Haemostas. 55, 24-32.

Mandelbaum, F. R. and AssakURA, M. T. (1988) Antigenic relationship of hemorrhagic factors and proteases isolated from the venoms of three species of Bothrops snakes. Toxicon 26, 379-385.

Mandelbaum, F. R., Assukura, M. T. and Reichl, A. P. (1984) Characterization of two haemorrhagic factors isolated from the venom of Bothrops neuwiedi. Toxicon 22, 193-206.

MiNTon, S. A. (1967) Observations on toxicity and antigenic makeup of venoms from juvenile snakes. In: Animal Toxins, pp. 211-222 (Russell, F. E. and Saunders, P. R., Eds). Oxford: Pergamon Press.

Rolim-Rosa, R., Vieira, E. G. J., Silfs-Villarroel, M., Siracusa, Y. Q. and Iizuka, H. (1980/81) Anàlise comparativa entre os diferentes esquemas de hiperimunização empregada na produção de soros antiofidicos pelo Instituto Butantan (1957-1979). Mem. Inst. Butantan 44/45, 259-270.

Rosenfeld, G. and KelEN, E. M. A. (1966) Cross neutralization of the coagulant activity of some snake venoms by antivenins. Toxicon $4,7-15$.

Siles-Villarroel, M., Furlanetto, R. S., Rolim-Rosa, R., Zelanti, F. and Navas, J. (1974) Contribuição ao estudo immunoquímico de venenos botrópicos. Mem. Inst. Butantan 38, 31-40.

Tu, A. T. and SAlafranca, E. S. (1974) Immunological properties and neutralization of the sea snakes venoms (II). Am. J. trop. med. Hyg. 23, 135-138. 